\title{
CrimRxiv
}

\section{Parks as crime inhibitors or generators:Examining parks and the role of their nearby context}

\author{
Adam Boessen, John R. Hipp
}

Published on: Jun 08, 2018

DOI: 10.21428/cb6ab371.9b624e26

License: Creative Commons Attribution 4.0 International License (CC-BY 4.0). 
